\title{
INTEGRAL CONSTRAINTS ON CONVECTIVE OVERSHOOTING TWO-DIMENSIONAL NUMERICAL STUDIES
}

\author{
IAN W. ROXBURGH AND JOHN SIMMONS \\ Astronomy Unit, Queen Mary and Westfield College, University of \\ London, Mile End Rd., London E1 4NS, UK.
}

\begin{abstract}
The integral constraint on convective overshooting (Roxburgh $1976,1978,1989$ ) is re-derived for for statistically stationary two-dimensional convection between stress-free horizontal boundaries maintained at constant temperatures, the vertical boundaries being periodic. We report on numerical simulations of such two-dimensional compressible convection in a fluid where the central regions are convectively unstable and the surrounding layers are stable. The results support the conclusion by Roxburgh (1989) that the contribution from the radiative flux is well approximated by the flux carried by the mean temperature gradient, but demonstrate that viscous dissipation is important, reducing the extent of overshooting.
\end{abstract}

\section{THE INTEGRAL CONSTRAINT}

We consider a compressible fluid confined between two horizontal stress free boundaries, $\mathrm{u}_{\mathrm{z}}=0$ at $\mathrm{z}=0, \mathrm{z}=\mathrm{h}$; the acceleration due to gravity is a constant $-\mathrm{g}$ in the vertical $\mathrm{z}$ direction, the top boundary is maintained at temperature $T_{1}$ and the bottom boundary at $T_{2}$ and the flow is two dimensional and is taken as periodic in the horizontal $x$ direction, $U(x, z, t)=U(x+a, z, t)$ for all variables $U$. Since $g_{i}=-\partial \Psi / \partial x i$ is independent of time the contribution to the entropy and total energy equations governing the fluid can be written in the form:

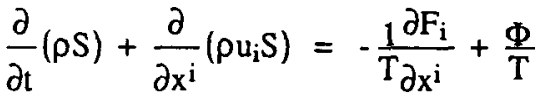

$$
\begin{aligned}
& \frac{\partial}{\partial t}\left[c_{v} \rho T+\frac{1}{2} \rho u^{2}+\rho \Psi\right]+\frac{\partial}{\partial x^{i}}\left[\rho u_{i}\left(c_{p} T+\frac{1}{2} u^{2}+\Psi\right)-u_{j} \eta_{i j}+F_{i}\right]=0 \\
& \Phi=\eta_{i j} \frac{\partial u_{i}}{\partial x^{j}}=\frac{1}{2} \eta\left(\frac{\partial u_{i}}{\partial x^{j}}+\frac{\partial u_{j}}{\partial x^{i}}-\frac{2}{3} \delta_{i j} \frac{\partial u_{k}}{\partial x^{k}}\right)^{2}
\end{aligned}
$$

with $\eta_{\mathrm{ij}}, \mathrm{F}_{\mathrm{i}}=-\mathrm{K}(\mathrm{p}, \mathrm{T}) \partial \mathrm{T} / \partial \mathrm{x}^{\mathrm{i}}, \mathrm{P}=R \mathrm{R} \mathrm{T} / \mu, \mathrm{E}=3 R \mathrm{~T} / 2 \mu, \mathrm{S}=(\mathcal{R} / \mu)$ ln $\left(\mathrm{T}^{3 / 2} / \rho\right)$, the viscous stress tensor, radiative flux, pressure, internal energy and entropy per unit mass. 
Let $<U>$ denote the time average of $U\left(x^{i}, t\right)$, then for statistically stationary convection $\langle\partial U / \partial t>=0$. Integrating the entropy and total energy equations over the volume of the fluid, using Gauss's theorem to convert integrals over the volume $\mathrm{V}$ to integrals over the surface $\Sigma$, and using the boundary conditions that $u_{z}=0, \eta_{x z}=0$ on $z=0, h$, and the periodic condition $U(x, z, t)=U(x+a, z, t)$, gives the two identities

$$
\int_{\Sigma}\left\langle F_{i}\right\rangle d \Sigma_{i}=0, \quad \int_{V}\left\langle\frac{1}{T}\left(\frac{\partial F_{i}}{\partial x^{i}}-\Phi\right)\right) d V=0
$$

The contribution to the surface integral from the vertical boundaries $(x=0, a)$ cancel by the periodic conditions, so the time averaged flux entering the bottom boundary equals that leaving the top boundary and is constant $\left(=a \Gamma_{0}\right)$. With $T$ constant on $z=0, h$, and periodic conditions on $x=0$, a, it follows that $\int\left\langle F_{i} / T>d \Sigma=a \Gamma_{0}\left(1 / T_{1}-1 / T_{2}\right)\right.$. Integrating the second of equation (4) by parts and using Gauss's theorem yields

$$
\int_{V}\left\langle\frac{1}{T} \frac{\partial F_{i}}{\partial x^{i}}\right\rangle d V=\int_{V}\left\langle\left(F_{i}-\Gamma_{0 i}\right) \frac{1}{T^{2}} \frac{\partial T}{\partial x^{i}}\right\rangle d V=\int_{V}\left\langle\frac{\Phi}{T}\right\rangle d V
$$

Defining the horizontal average $\overline{U(z, t)}=(1 / a) \int U d x$ and $\left.U_{0}(z)=\overline{(U(z, t)}\right\rangle$ then integrating over the horizontal direction gives the integral constraint in the form

$$
\int_{0}^{h} \overline{\left.\left(F_{i}-\Gamma_{0 i}\right) \frac{1}{T^{2}} \frac{\partial T}{\partial x^{i}}\right\rangle} d z=\int_{0}^{h} \overline{\left\langle\frac{\Phi}{T}\right\rangle} d z>0
$$

Let $V(z)=\overline{\Phi / T}$ and $I_{1}(z)=\overline{\left(F_{i}-\Gamma_{i}\right)(1 / T)^{2} \partial T / \partial x^{i}}$. In Roxburgh (1978), $I_{1}(z)$ was replaced by $I_{0}(z)=\left(F_{0}-\Gamma_{0}\right)\left(1 / T_{0}\right)^{2} d T_{0} / d z$, calculated using the mean values of $T$ and the flux given by the mean field. This was criticised by Baker and Kuhfuß (1987) on the grounds that it neglected terms quadratic in the fluctuating quantities, but Roxburgh (1989) argued that these contributions are expected to be small if the mean stratification is almost adiabatic. In the numerical simulations reported here the difference between $I_{0}$ and $I_{1}$ is indeed small, but the viscous dissipation $V(z)$ is not small and cannot be neglected.

\section{NUMERICAL RESULTS}

Numerical simulations were made for a layer in which the variation of the conduction coefficient with temperature was $K(T)=K o\left(\alpha T^{5}+\beta / T^{3}\right)$, with $(\alpha, \beta)$ chosen so that the central regions of the layer are convectively unstable and the surrounding regions stable; the temperature at the bottom of the layer was twice that at the top and the viscosity was constant. The equations were integrated for 50 times the turn over time of the convection using a 64×64 array machine (DAP). A running time average was calculated at each mesh point and a horizontal average calculated at the end of a computing run. The integrands $I_{0}(z), I_{1}(z)$ and $V(z)$ that enter the integral constraint were then determined. 
Figure 1 shows the flow pattern for a case where the Rayleigh number at the centre of the layer $R_{m}=120 R_{c}$, where $R_{c}$ is the value at the onset of convection, and the Prandil number $\sigma=\eta c_{p} / K=1$. Figure 2 shows the values of the integrands $I_{0}(z)$ and $I_{1}(z)$ and the viscous dissipation term $V(z)$, Figure 3 gives results for $\sigma=0.1$

As can be seen from these figures $I_{1}=I_{0}$, and the contribution from terms quadratic in the fluctuating quantities is therefore small. The Integral Constraint is well approximated by

$$
\left.\int_{0}^{h}\left(F_{0}-\Gamma_{0}\right) \frac{1}{T_{0}^{2}} \frac{d T_{0}}{d z} d z=\int_{0}^{h} \bar{T} \frac{\Phi}{T}\right\rangle d z
$$

For the cases $\sigma=0.1,1$, studied here viscous dissipation cannot be neglected, although perhaps there is some indication that it may be less important for small $\sigma$ ( $\sigma=10^{-6}$ in stars), but further calculations are needed on this point. Since $\mathrm{V}>0$. neglecting viscous dissipation can provide an upper limit on the extent of convective overshooting.

\section{ACKNOWLEDGEMENTS}

The authors gratefully acknowledge support from SERC; tWR for grant GR/H 09454, and IS for an SERC studentship.

\section{BEFERENCES}

Baker, N H and KuhfuB, R., 1987. 'On the Roxburgh Criterion for Convective Overshooting', Astronomy and Astrophysics, 185, pp 117-123.

Roxburgh, I W, 1976, 'The Internal Structure of the Sun and Solar Type Stars', in Basic Mechanisms of Solar Activity, ed V Bumba and J Kleczeck, D Reidel, pp 451-456.

Roxburgh, I W, 1978, 'Convection and Stellar Structure', Astronomy and Astrophysics, 65, pp 281-285

Roxburgh, I W, 1989, 'Integral Constraints on Convective Overshooting', Astronomy and Astrophysics, 211, pp 361-364 